\title{
On mean coordination and structural heterogeneity in model amorphous solids
}

Cite as: J. Chem. Phys. 132, 024906 (2010); https://doi.org/10.1063/1.3284786

Submitted: 07 May 2009 . Accepted: 14 December 2009 . Published Online: 11 January 2010

Alessio Zaccone, and Emanuela Del Gado

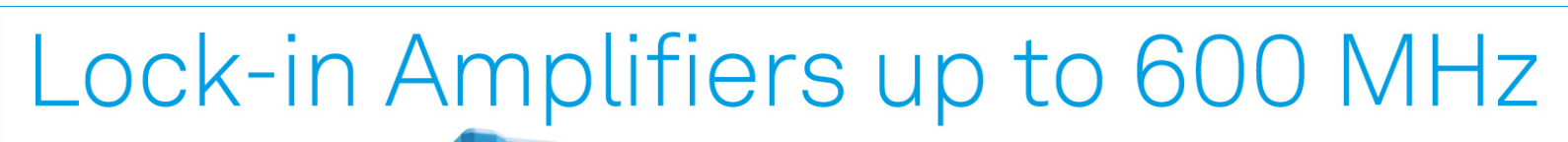

starting at

$$
\$ 6,210
$$

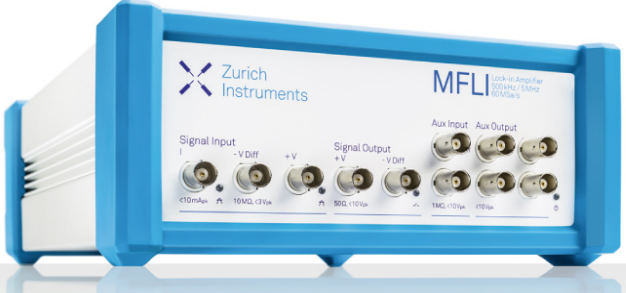

J. Chem. Phys. 132, 024906 (2010); https://doi.org/10.1063/1.3284786

(c) 2010 American Institute of Physics.
132,024906 


\title{
On mean coordination and structural heterogeneity in model amorphous solids
}

\author{
Alessio Zaccone ${ }^{1, a)}$ and Emanuela Del Gado ${ }^{2}$ \\ ${ }^{1}$ Chemistry and Applied Biosciences, ETH Zurich, CH-8093 Zurich, Switzerland \\ ${ }^{2}$ Polymer Physics, ETH Zurich, CH-8093 Zurich, Switzerland
}

(Received 7 May 2009; accepted 14 December 2009; published online 11 January 2010)

\begin{abstract}
We propose a simple route to analytically evaluate the average coordination of model disordered solids with maximally homogeneous distribution of the particles in space. The model yields the average number of contacts $(z)$ as a function of volume fraction $(\phi)$ of a hard-sphere connected system and recovers the critical jamming point of hard spheres $(z=6$ at $\phi=0.64)$. Numerical simulations of Lennard-Jones glasses with a varying attraction range are used to investigate the volume fraction dependence of the average coordination in the presence of attraction. It is observed that upon decreasing $\phi$ below 0.6 , structural heterogeneity is reflected in values of the coordination number which are higher than those predicted by the model for a statistically homogeneous distribution of particles in space due to the attraction-induced local aggregation. Thus the model can be usefully employed as a quantitative reference to assess the degree of structural heterogeneity in glasses in terms of a directly accessible structural parameter such as the mean number of contacts. (C) 2010 American Institute of Physics. [doi:10.1063/1.3284786]
\end{abstract}

\section{INTRODUCTION}

Dynamical arrest in amorphous condensed and soft condensed matter represents an open problem where the arising of rigidity is closely associated with subtle structural changes. ${ }^{1-3}$ Therefore, it is essential to define measures in order to properly describe the structure as well as to follow its transformations. The simplest topological parameter, which is used to describe the structure of amorphous condensed matter, is the mean coordination number. ${ }^{1}$ Its definition does not gather general consensus and highly depends upon the system under consideration. From the experimental viewpoint, structural information can be obtained by either radiation scattering or by optical (e.g., microscopy) techniques (which can be applied, for example, to colloidal and granular systems). While scattering provides structural information in Fourier space, microscopy and direct imaging give quantitative, real space information about the local coordination. A practical way to define a mean coordination number is to identify it with the mean number of contacts, i.e., counting the number of particles physically touching a tagged one. ${ }^{4}$ Some uncertainty may still arise as to the degree of "touching" between particles, so that some arbitrariness is unavoidable. ${ }^{4}$ From the theoretical standpoint, most theories of condensed matter structure produce as a result the familiar radial distribution function (rdf), $g(r){ }^{5}$ Integrating $g(r)$ over a value of shell width around the tagged particle gives the most likely number of particles to be found within that radial distance. Also in this case, however, different choices are possible for the integration boundary and this introduces again a substantial degree of arbitrariness in the characterization of the local structure. ${ }^{5}$

${ }^{a)}$ Electronic mail: alessio.zaccone@chem.ethz.ch.
Besides these problems, there is the issue of characterizing the structure of amorphous systems at a more coarsegrained level, where the appearance of structural heterogeneity usually plays an important role. ${ }^{2}$ This has been done, for example, in terms of the statistics and structure of the voids, including remoteness and the void size distribution, which have been usefully employed in simulation studies of fractal colloidal aggregates and gels as well as colloidal glasses. ${ }^{6,7}$ In this work we suggest the possibility to give a description of structural heterogeneity in terms of the mean coordination number, which is a more accessible quantity. We also use the mean coordination number to assess the effect on structure (and structural heterogeneity) of different microscopic interaction potentials (attractive rather than merely repulsive).

To this aim, we focus our attention on amorphous model (colloidal ${ }^{8}$ and Lennard-Jones) systems. We start by proposing an analytic formula to estimate the mean number of contacts $(z)$ in purely hard-sphere connected (jammed) systems with homogeneous spatial distribution of the particles. Then we consider the arising of structural heterogeneity as due to the addition of an attractive component of interaction in simulated Lennard-Jones glasses. In both cases, we estimate $z$ via the number of neighbors at different volume fractions $\phi$ and compare it with the theoretical predictions for the homogeneous system. On the basis of such comparisons we propose a criterion to indirectly assess the presence of structural heterogeneity in terms of the deviation of the measured $z(\phi)$ from the theoretical prediction for homogeneous systems.

\section{MODEL}

A deep and rapid quench of a supercooled liquid is able to freeze in the liquid structure almost instantaneously, so that the resulting (solid) glassy state presents a spatial organization which cannot be distinguished, in practice, from that 
of the liquid snapshot at the quenching time. In the theoretical framework proposed by Alexander, ${ }^{9}$ this would correspond to the assumption that the set of interparticle distances in the rigid reference glass state $\{\mathbf{R}\}$ is the same or approximately the same as the set of interparticle distances in the liquid snapshot $\{\mathbf{r}\}$ at the quenching time $t_{q}$,

$$
\{\mathbf{R}\}_{\text {glass }} \approx\left\{\mathbf{r}\left(t_{q}\right)\right\}_{\text {liquid }} \text {. }
$$

In Alexander's approach, it is clear that the situation is more complicated than this simplistic picture because the particle positions in the liquid snapshot correspond to an unstable structure which is out of mechanical equilibrium. ${ }^{9}$ Even for an ideal, infinitely fast quench, where diffusive motion is suppressed, a solidlike relaxation process accompanies the quench and is responsible for the creation, through a hierarchy of restructuring and buckling phenomena, of the mechanically stable structure which characterizes the final amorphous solid. ${ }^{9}$ The theoretical description of such complex structural reorganization phenomena is out of reach at present. What we attempt to do in the following is to find an analytical route which, starting from the liquid structure, leads to the number of contacts in the final arrested state.

The simplest case one can think of is that of a liquid of hard spheres (where the interaction reduces to mutual impenetrability at contact). Upon quenching (at zero applied pressure) there is a unique $T=0$ state, which is mechanically (marginally) stable and corresponds to the jamming point of hard spheres at volume fraction $\phi \approx 0.64$, denoted as point $J$, where (marginal) rigidity is expressed through the isostatic condition $z=2 d=6$, where $z$ is the mean coordination and $d=3$ is the dimensionality of space. It was shown already by Maxwell that a structure possesses rigidity when the total number of degrees of freedom is at least equal to the total number of constraints $N_{c}$. That is, for a system of $N$ particles, $N_{c} \geq 3 N-6 .{ }^{10}$ As shown in recent literature, point $J$ displays the properties of a critical point where the Maxwell criterion is only marginally satisfied (isostaticity), and for large systems one thus has $z=2 N_{c} / N=6 .{ }^{11}$ At volume fractions lower than point $J$, the quenched metastable states are usually very tenuous at $T>0$ (and mechanically unstable at $T=0$ ), since the elasticity of hard-sphere glasses then is exclusively entropic and due to particle caging. ${ }^{1}$ In the presence of an attractive interaction, substantially rigid states can be instead obtained even at $\phi \ll 0.64 .^{12}$ In the latter case, however, the extent of attractive interaction also affects the spatial organization favoring the appearance of structural heterogeneity. ${ }^{12}$

Here we start by deriving an accurate analytical approximation for the short-range part of the rdf of dense hardsphere liquids which represent the most homogeneously disordered system known. Integrating the liquid rdf over a certain value of shell width may certainly give a flavor of how the probability of finding neighbors at that distance from the tagged particle changes as a function of density, but does not correspond to any well-defined quantity from a physical point of view. Instead we submit the liquid snapshot structure to a so-called "hyperquenching" protocol. ${ }^{11}$ This may be visualized as the process of expanding the particle size in the liquid snapshot until the particles come into contact with each other and each particle gains on average a certain finite number of contacts. An essential constraint to be satisfied is that the system must be an isostatic random close packing at point $J$, the jamming point of hard spheres. $^{13}$

Mathematically, such hyperquenching protocol can be realized by integrating the rdf of the hard-sphere liquid with an integration boundary which allows to recover point $J$ at $\phi=0.64$. The result of the integration with the boundary determined in this way is an estimate of the mean contact number $z$ as a function of the volume fraction for the arrested homogeneous hard-sphere system, which recovers the isostatic packing at $\phi=0.64$, i.e., point $J$.

The structure of a (disordered) fluid of $N$ particles with diameter $\sigma$ is entirely described via the $n$-particle distribution function defined by

$$
g_{N}^{(n)}\left(\mathbf{r}^{n}\right)=\frac{\rho_{N}^{(n)}\left(\mathbf{r}_{1}, \ldots, \mathbf{r}_{n}\right)}{\prod_{i=1}^{N} \rho_{N}^{(1)}\left(\mathbf{r}_{i}\right)} .
$$

For a uniform system $\rho_{N}^{(1)}(\mathbf{r})=N / V=\rho$, i.e., the number density of the fluid. If the system is homogeneous and isotropic, we have $g_{N}^{(2)}\left(\mathbf{r}_{1}, \mathbf{r}_{2}\right)=g(r)$, i.e., the rdf. The delta function representation of the rdf gives clear evidence of its geometrical meaning in terms of (random) correlations between particles positioned around a reference one, ${ }^{5}$

$$
\rho g(r)=\frac{\rho^{2}}{N} \int g_{N}^{(2)}\left(\mathbf{r}, \mathbf{r}^{\prime}\right) d \mathbf{r}^{\prime}=\left\langle\frac{1}{N} \sum_{i=1}^{N} \sum_{j=1}^{N} \delta\left(\mathbf{r}-\mathbf{r}_{j}+\mathbf{r}_{i}\right)\right\rangle .
$$

The direct correlation function $c(r)$ is defined by the Ornstein-Zernike convolution relation $h(r)=c(r)+\rho \int h\left(r^{\prime}\right)$ $\times c\left(\left|\mathbf{r}-\mathbf{r}^{\prime}\right|\right) d \mathbf{r}^{\prime}$ which can be solved within the PercusYevick (PY) closure, $c(r) \approx\left[1-e^{\beta U(r)}\right] g(r)$, where $U$ is the interaction potential, the solution being piecewise analytic. ${ }^{5}$ Since we are interested in the near-contact region, we can approximate the PY solution in the range of $1 \leq r / \sigma \leqq 1.1$ using the following formula: ${ }^{14}$

$$
\begin{aligned}
g^{\prime}\left(r / \sigma^{\prime} ; \phi^{\prime}\right)= & \left(1+\phi^{\prime} / 2\right) /\left(1-\phi^{\prime}\right)^{2} \\
& -(9 / 2) \phi^{\prime}\left(1+\phi^{\prime}\right) /\left(1-\phi^{\prime}\right)^{3}\left(r / \sigma^{\prime}-1\right) .
\end{aligned}
$$

Correcting for the contact value at high density and for the phase shift according to the Verlet-Weis prescription leads to 5

$$
g(r / \sigma ; \phi)=g^{\prime}\left(r / \sigma^{\prime} ; \phi^{\prime}\right)+\delta g_{1}(r / \sigma),
$$

where $\phi^{\prime} \approx \phi-\phi^{2} / 16$ and $\sigma^{\prime}=\sigma\left(\phi^{\prime} / \phi\right)^{1 / 3}$. The short-range term can be expressed as

$$
\delta g_{1}(r / \sigma)=\delta g_{1}(x)=(A / x) \exp [-\alpha(x-1)] \cos [\alpha(x-1)],
$$

where $x=r / \sigma$, and $A$ and $\alpha$ are coefficients which are only functions of $\phi$ and contain the contact value of the rdf, $g(1 ; \phi)$. The advantage of this formulation is that the contact behavior of the PY solution can be modulated by setting $g(1 ; \phi)$ equal to the value predicted by equations of state valid in the high-density regime, such as the Hall, as well as by numerical simulations. Equations (4)-(6), as can be easily 


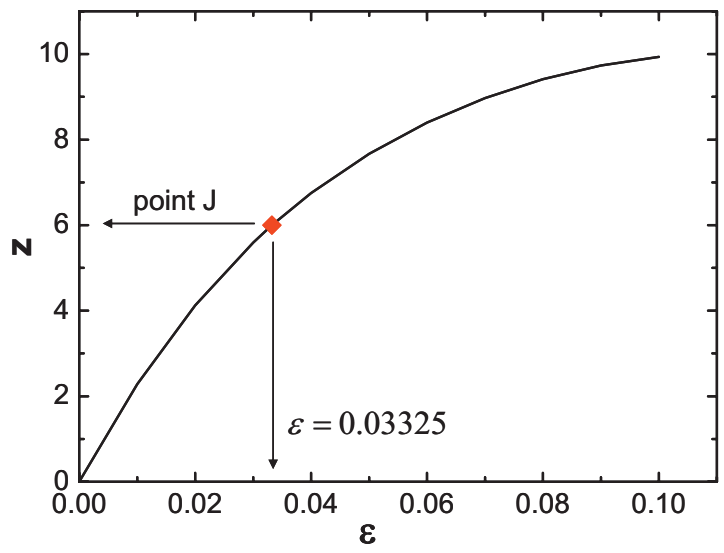

FIG. 1. The contact number in the hyperquenched system as a function of the parameter $\varepsilon$ calculated from Eqs. (8) and (9) for $\phi=0.64$. The diamond identifies the value of the parameter $\varepsilon$ which recovers the isostatic jamming point of hard spheres $(z=6)$, that is $\varepsilon=0.03325$.

verified, are in excellent agreement with the most recent exact rdf from computer simulations in the range of $1 \leq x$ $\lesssim 1.1$. $^{15}$ However, it can be noted from the above equations that the rdf is given exclusively as a function of geometrical parameters, independent of temperature.

The integral of $\rho g(r)$ over a volume element $d^{3} r$ is just the number of atoms in that volume element and volume integration yields the total number of atoms minus the one at the origin, $\int \rho g(\mathbf{r}) d^{3} r=N-1$. The volume integration of $\rho g(r)$ for isotropic systems

$$
z=\rho \int_{\sigma}^{r} 4 \pi r^{2} g(r) d r=24 \phi \int_{1}^{x} x^{2} g(x) d x
$$

gives the number of particles $z$ in a shell of thickness $r-\sigma$ (or $x-1$ ) around a given particle.

Seen from a different perspective, the integral in Eq. (7) represents a mapping between the original liquid state, described by $g(r)$, and a state with a mean number of contacts given by $z$. The latter state is obtained by expanding the size of the original particles in the liquid snapshot by an amount such that each particle ends up being, in the final state, in physical contact with $z$ neighbors. We can schematize the hyperquenching in two steps.

(i) Quench the dense (hard-sphere) liquid instantaneously so that thermal motion is suppressed and the liquid snapshot structure is frozen in.

(ii) Increase the particle diameter from $\sigma$ to $\hat{\sigma}=\sigma+\varepsilon \sigma$, where $\hat{\sigma}$ is the sphere diameter in the jammed configuration while $\varepsilon$, the hyperquenching parameter, is a (small) number.

What one obtains from step (i) is clearly a configuration corresponding to the frozen-in liquid snapshot. Then, in step (ii), the reference particle comes into contact with a number of nearest neighbors which depends upon the value of $\varepsilon$. Note that since collisions among particles may occur in the liquid, at step (i) some particles may already be at contact. This requires displacing the contacted particles by an amount $\varepsilon \sigma$ at step (ii). Such an effect can be ignored if the $\varepsilon$ value is small in comparison to $\sigma$.
We thus proceed by first constructing the formula which yields the nearest-neighbors number in the liquid as a function of the dimensionless gap (or shell width) and volume fraction, and subsequently submit it to the hyperquenching procedure. The integral in Eq. (7) can be rewritten in the interparticle gap $l=(r-\sigma) / \sigma=x-1 \quad$ as $z(\varepsilon ; \phi) \equiv 24 \phi \int_{0}^{\varepsilon}$ $\times(1+l)^{2} g(l) d l$. Then, applying Eqs. (4)-(6), one can integrate analytically and we arrive at the following expression:

$$
\begin{aligned}
z(\varepsilon ; \phi) \equiv & 24 \phi \int_{0}^{\varepsilon}(1+l)^{2} g(l) d l \\
= & 2 \phi\left\{\varepsilon \left[4 \frac{1+\phi^{\prime} / 2}{\left(1-\phi^{\prime}\right)^{2}}\left(3+3 \varepsilon+\varepsilon^{2}\right)\right.\right. \\
& \left.-\frac{9}{2} \phi^{\prime} \frac{1+\phi^{\prime}}{\left(1-\phi^{\prime}\right)^{3}} \varepsilon\left(6+8 \varepsilon+3 \varepsilon^{2}\right)\right] \\
& +6(A / \alpha) \exp (-\alpha \varepsilon)[\alpha \exp (-\alpha \varepsilon) \\
& +\sin \alpha \varepsilon+\alpha(1+\varepsilon)(\sin \alpha \varepsilon-\cos \alpha \varepsilon)]\},
\end{aligned}
$$

which gives the number of nearest neighbors in the liquid within the dimensionless gap $\varepsilon$. Since we are interested in the high-density regime, we can evaluate the Verlet-Weis parameters, $A$ and $\alpha$, using the contact value of the rdf, $g\left(1 ; \phi^{\prime}\right)$, from the Hall equation of state for the fluid branch of the high-density hard-sphere system (which employs up to seven virial coefficients and is thus valid in the high-density regime), ${ }^{16}$ and we obtain

$$
\begin{aligned}
A= & (3 / 4) \phi^{\prime 2}\left(1-0.7117 \phi^{\prime}-0.114 \phi^{\prime 2}\right) /\left(1-\phi^{\prime}\right)^{4} \\
& -\left[1-\left(\phi^{\prime} / 2\right)\right] /\left(1-\phi^{\prime}\right)^{3}+0.4074\left(\phi^{\prime}-0.9378\right) \\
& \times\left(1.3948-0.7365 \phi^{\prime}+\phi^{\prime 2}\right) \times\left(1.7988+1.9685 \phi^{\prime}\right. \\
& \left.+\phi^{\prime 2}\right) /\left[\left(-0.7405+\phi^{\prime}\right)\left(1.2947-2.1357 \phi^{\prime}+\phi^{\prime 2}\right)\right],
\end{aligned}
$$

$$
\begin{aligned}
\alpha= & 18\left(1-0.7117 \phi^{\prime}-0.114 \phi^{\prime 2}\right) /\left(1-\phi^{\prime}\right)\left[1-\left(\phi^{\prime} / 2\right)\right] \\
& \times\left\{\left[1-\left(\phi^{\prime} / 2\right)\right] /\left(1-\phi^{\prime}\right)^{3}\right\} /\left\{0.4074\left(\phi^{\prime}-0.9378\right)\right. \\
& \times\left(1.3948-0.7365 \phi^{\prime}+\phi^{\prime 2}\right) \times\left(1.7988+1.9685 \phi^{\prime}\right. \\
& \left.\left.+\phi^{\prime 2}\right) /\left[\left(-0.7405+\phi^{\prime}\right)\left(1.2947-2.1357 \phi^{\prime}+\phi^{\prime 2}\right)\right]\right\} .
\end{aligned}
$$

At this point, we can fix the hyperquench by assigning to $\varepsilon$ a value which recovers the critical point $J$ of hard spheres. Thus, from the latter condition we find that $z=6$, i.e., the isostatic solid is obtained at $\phi=0.64$, if one sets

$$
\varepsilon=0.03325 \text {. }
$$

This fixes the hyperquenching protocol in that now Eqs. (8)-(10) produce a unique $z(\phi)$ curve which passes through point $J$ (i.e., through $z=6$ at $\phi=0.64$ ). For illustration we have plotted the dependence of $z$ upon $\varepsilon$ at $\phi=0.64$ in Fig. 1.

In the presence of attraction, we may expect two distinct scenarios depending upon the volume fraction. In the first, density is high enough that restructuring driven by attraction is strongly hindered (frustration), so that the structure is spatially homogeneous and a mean coordination number for the contacts close to the value predicted by Eqs. (8)-(10) should 


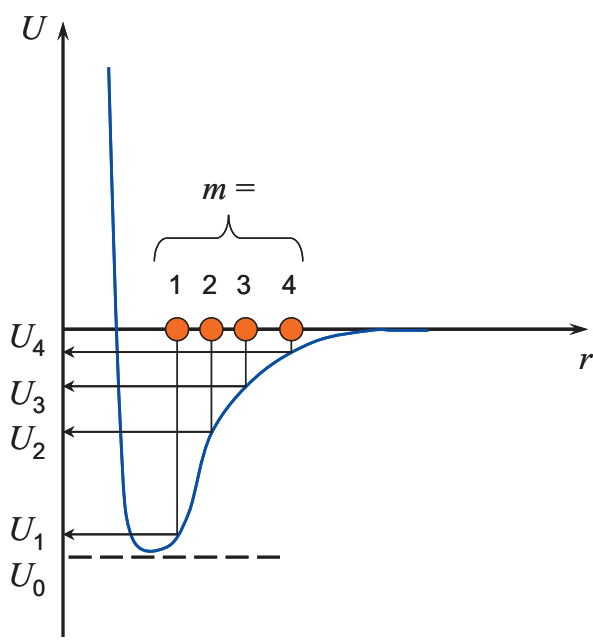

FIG. 2. Schematic representation of an interaction potential with attraction (such as, e.g., for Lennard-Jones systems). The arrows indicate the energy levels corresponding to the positions on the radial distance axis occupied by the neighbors. See text and Eq. (11).

be expected at the end of the quench. In this case, $z<6$, the system is rigid due to initial stresses and harmonically restoring forces. ${ }^{9}$ In the second scenario, density is not high enough to forbid larger scale restructuring and aggregation of the particles into locally denser regions (clusters), accompanied by a reduction in the internal energy of the system. Therefore when structural heterogeneity becomes important, the mean coordination is expected to be higher than the model prediction.

In order to test these speculations, also from a quantitative point of view, as well as the model itself, we have carried out simulations on model glassy systems with attraction.

\section{MOLECULAR DYNAMICS SIMULATIONS OF LENNARD-JONES GLASSES}

Molecular dynamics (MD) simulations have been carried out on a binary mixture of 2024 atoms with size ratio of 1:1.2 interacting via two different cutoff Lennard-Jones-type potentials with exponents $20-10$ and 40-20. The system was first driven into the deepest metastable minimum in the energy landscape at $T \approx 0.46$. Three different glass configurations for each potential obtained in this way were then quenched to $T=0.005$ for $10^{6} \mathrm{MD}$ steps. Further, the simulations provide an indication of the mean local structure of the glass. A readily accessible quantity in this respect is provided by the average internal energy per particle, $\left\langle E_{\text {int }}\right\rangle$.

For systems with an attractive component of interaction, there is a direct relationship between mean coordination and the macroscopic elastic response, as shown also in recent work. ${ }^{11}$ Attraction provides indeed restoring forces which can oppose an externally applied deformation, thus conferring rigidity to the system. It seems natural then to identify the mean coordination number with the number of mechanical contacts which are involved in the restoring forces. It is likely that the neighbors that give a major contribution to stress bearing are those localized near the minimum of the potential well. In fact, in the Cauchy-Born approach for amorphous solids by Alexander, ${ }^{9}$ if one neglects nonaffine
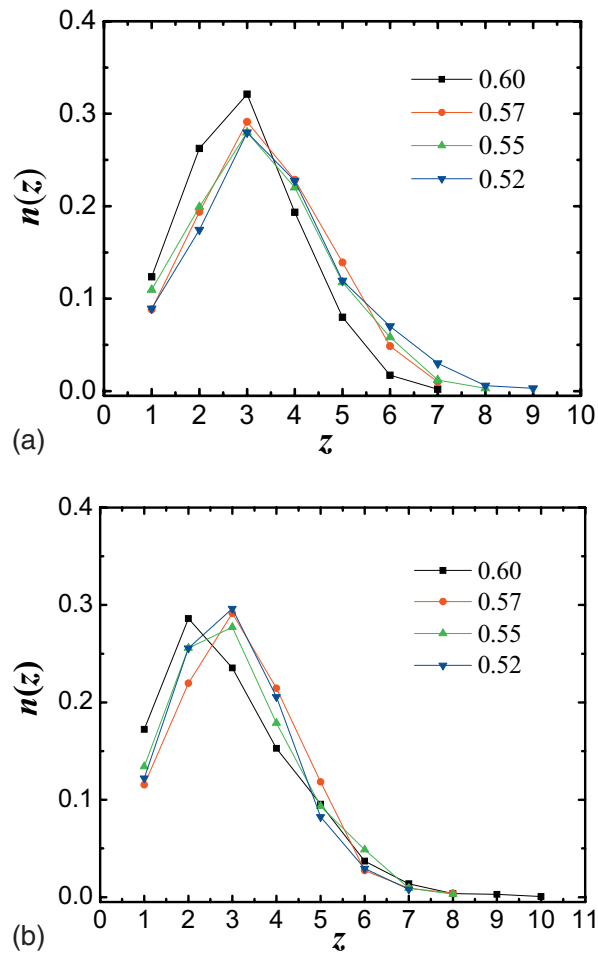

FIG. 3. Fraction of particles having a number of neighbors $z$ within the minimum of the potential well at different volume fractions (see legend). (a) Lennard-Jones 20-10 system. (b) Lennard-Jones 60-30 system.

relaxations (which are usually a small contribution for strongly bonded glasses) the dominant term in the free energy expansion is the one involving the second derivative of the interaction potential evaluated at the minimum of the potential well. The neighbors, which are positioned in the low-energy tail of the potential, instead, are expected to play a negligible role in the stress-bearing mechanism. Therefore we calculate the mean number of contacts in glasses with attraction

$$
z \equiv \frac{1}{N} \sum_{l=1}^{N} \sum_{m=1}^{n n} w_{m}^{(l)}=\frac{1}{N} \sum_{l=1}^{N} \sum_{m=1}^{n n} U_{m}^{(l)} / U_{0},
$$

i.e., for each particle $l$ the number of mechanical contacts is determined as the weighted average number of its neighbors where for each $m$ th neighbor of $l$ the weight $w_{m}^{(l)}$ is given by its interaction potential energy level, $U_{m}^{(l)}$, normalized by the depth of the potential well, $U_{0} . n n$ is the number of neighbors around the $l$ th particle. In the case of truncated LennardJones potentials, as they are often used in simulations, this is just the number of particles within the cutoff distance. For a schematic illustration of the terms in Eq. (11), see Fig. 2. The expression Eq. (11) corresponds to the internal energy per particle $\left\langle E_{\text {int }}\right\rangle$, as calculated from our MD simulations.

\section{RESULTS AND DISCUSSION}

In Fig. 3 distributions for the fraction of particles $n(z)$ within a distance equal to the minimum of the potential well having coordination number $z$ are shown for the two Lennard-Jones potentials investigated. We observe that in both cases the distribution broadens toward higher values of 


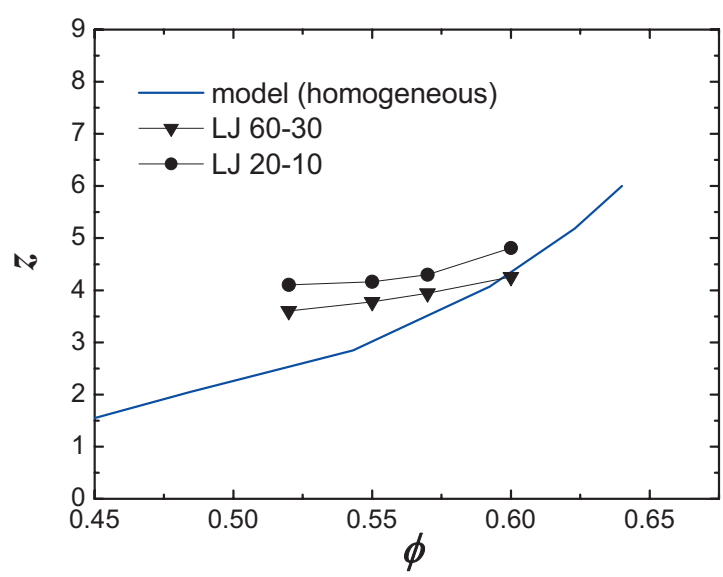

FIG. 4. Comparison between the contact number as a function of volume fraction curve calculated from Eqs. (8)-(10) (solid line) and the contact numbers evaluated from simulations of Lennard-Jones glasses according to Eq. (11) (symbols).

$z$ upon lowering the volume fraction below $\phi=0.6$. In particular, in the case of the $20-10$ potential the distribution clearly develops a tail at high values of $z$ which tends to grow upon lowering the volume fraction. This indicates that at lower volume fractions, the geometric frustration being less severe, particles can more easily rearrange during the quenching process to aggregate locally with a significantly higher value of $z$ (nearly by a factor of 2).

It is clear that for this reason structural heterogeneity must increase upon lowering the volume fraction. Therefore, one can describe the arising of structural heterogeneity upon decreasing $\phi$ in terms of the formation of locally very dense regions (clusters) characterized by a coordination number significantly higher.

In Fig. 4 we report the theoretical $z(\phi)$ curve and the average values for the Lennard-Jones glasses evaluated according to Eq. (11). We observe that the $z$ values for the Lennard-Jones systems are rather close to the theoretical predictions around $\phi=0.6$ (where indeed we could not see signatures of structural heterogeneity in Fig. 3), but then decrease much less rapidly upon decreasing $\phi$ and become increasingly higher with respect to the theoretical prediction. Based on what suggested by the analysis of Fig. 3, this can be explained if one thinks that upon decreasing $\phi$ the locally denser regions still retain a very high value of contact number and make an important contribution to the measured sample-averaged value even at the lowest $\phi$. A much weaker dependence of the average $z$ upon $\phi$, as compared with the perfectly spatially homogeneous case, and the consequent higher values of $z$, could be then interpreted as hallmarks of significant structural heterogeneity. The deviation increases upon decreasing $\phi$ as the structural heterogeneity becomes more important.

This suggests that the $z(\phi)$ curve of a specific glassy system can be used to assess the extent of structural heterogeneity in the system by comparing it with the theoretical curve, Eqs. (8)-(10), corresponding to the highest degree of structural homogeneity. The structural heterogeneity is ex- pected to be more pronounced the more the $z(\phi)$ curve will depart from the theoretical one.

\section{CONCLUSION}

We derived an analytical formula (using liquid theory and the properties of the jamming point of hard spheres) to estimate the mean number of contacts in hard-sphere systems as a function of the volume fraction. The predictions apply whenever the distribution of the particles in space is homogeneously disordered. Furthermore, we have investigated the contact number versus volume fraction in model amorphous solids where a significant attractive component of the interaction is present (Lennard-Jones glass). In these cases we estimate the average contact number from the internal energy per particle. In the measured distributions of the contact number, we found clear signatures of the development of structural heterogeneity upon lowering the volume fraction corresponding to a significant deviation from the homogeneous curve for $\phi<0.6$. At higher $\phi$, geometric frustration due to packing constraints overwhelms the tendency to develop heterogeneity induced by attraction and the theoretical estimate may have a universal validity (with spherical particles) independent of the microscopic interaction potential.

In conclusion, we have proposed an effective method to assess the structural heterogeneity in disordered media with spherical particles, in terms of the comparison of the mean contact number measured as a function of $\phi$, and a theoretical curve obtained for homogeneous systems. Based on the cases examined, we suggest that observed values of $z$ larger than the theoretical ones indicate a significant structural heterogeneity, which usually grows upon further lowering the volume fraction.

\section{ACKNOWLEDGMENTS}

Dr. Hua Wu is gratefully acknowledged for many helpful suggestions and discussions.

\footnotetext{
${ }^{1}$ K. Binder and W. Kob, Glassy Materials and Disordered Solids: An Introduction to Their Statistical Mechanics (World Scientific, Singapore, 2005).

${ }^{2}$ P.-G. de Gennes, C. R. Phys. 3, 1263 (2002).

${ }^{3}$ L. Cipelletti and L. Ramos, J. Phys.: Condens. Matter 17, R253 (2005).

${ }^{4}$ T. Aste, M. Saadatfar, and T. J. Senden, Phys. Rev. E 71, 061302 (2005).

${ }^{5}$ J. P. Hansen and I. R. McDonald, Theory of Simple Liquids (Academic, New York, 1986).

${ }^{6}$ M. D. Haw, Soft Matter 2, 950 (2006).

${ }^{7}$ R. M. L. Evans and M. D. Haw, Europhys. Lett. 60, 404 (2002).

${ }^{8}$ H. Tanaka, S. Jabbari-Farouji, J. Meunier, and D. Bonn, Phys. Rev. E 71, 021402 (2005).

${ }^{9}$ S. Alexander, Phys. Rep. 296, 65 (1998).

${ }^{10}$ J. C. Maxwell, Philos. Mag. 27, 294 (1864).

${ }^{11}$ M. Wyart, Ann. Phys. (Paris) 30, 1 (2005); A. Zaccone, H. Wu, and E. Del Gado, Phys. Rev. Lett. 103, 208301 (2009).

${ }^{12}$ M. Laurati, G. Petekidis, N. Koumakis, F. Cardinaux, A. B. Schofield, J. M. Brader, M. Fuchs, and S. U. Egelhaaf, J. Chem. Phys. 130, 134907 (2009)

${ }^{13}$ C. S. O’Hern, L. E. Silbert, A. J. Liu, and S. R. Nagel, Phys. Rev. E 68, 011306 (2003).

${ }^{14}$ D. Henderson and E. W. Grundke, J. Chem. Phys. 63, 601 (1975).

${ }^{15}$ A. Trokhymchuk, I. Nezbeda, J. Jirsák, and D. Henderson, J. Chem. Phys. 123, 024501 (2005).

${ }^{16}$ K. R. Hall, J. Chem. Phys. 57, 2252 (1972).
} 